\title{
Twin-peak quasiperiodic oscillations as an internal resonance (Research Note)
}

\author{
J. Horák and V. Karas
}

Astronomical Institute, Academy of Sciences, Boční II, 14131 Prague, Czech Republic

e-mail: vladimir.karas@cuni.cz; horak@astro.cas.cz

Received 12 August 2005 / Accepted 30 December 2005

ABSTRACT

Two peaks occur in high-frequency power spectra of X-ray lightcurves of several black-hole candidates. We further explore the idea that a non-linear resonance mechanism operating in strong-gravity regime is responsible for these inter-related quasi-periodic oscillations (twin QPOs). By extending the multiple-scales analysis of Rebusco, we construct two-dimensional phase-space sections, which enable us to identify different topologies governing the system and to follow the evolutionary tracks of the twin peaks. This approach suggests that the original (Abramowicz \& Kluźniak) parametric-resonance scheme can be viewed as a naïve account of the QPOs model with an internal resonance.We show an example of internal resonance in a system with up to two critical points, and describe a general technique that permits other cases to be treated in a systematical manner. A separatrix divides the phase-space sections into regions of different topology. Inside the libration region, the evolutionary tracks bring the observed twin-peak frequencies to an exact rational ratio, whereas the observed frequencies remain off resonance in the circulation region. Our scheme predicts the power should be cyclically exchanged between the two oscillations. Like the high-frequency QPOs in neutron-star binaries, one also expects, as a general property of the non-linear model, that in black-hole sources slight detuning pushes the twin-peak frequencies out of sharp resonance.

Key words. accretion, accretion-discs - black hole physics

\section{Introduction}

Twin peaks occur in high-frequency $(\sim 50-450 \mathrm{~Hz})$ power spectra of the X-ray ( 2-60 keV) lightcurves of several black-hole candidates; see van der Klis (2006), McClintock \& Remillard (2006) for recent reviews of observational properties and theoretical interpretations. This transient phenomenon seems to be connected to the kilohertz quasi-periodic oscillations (QPOs) in neutron star sources, of which more examples are known (in the tens at present). The nature of black-hole high-frequency QPOs remains puzzling despite the variety of models proposed in the literature. In neutron-star low-mass X-ray binaries, these twin QPOs are known to often occur simultaneously. They can be highly coherent ( $Q \gtrsim 10^{2}$; e.g. Barret et al. 2005), while slowly wandering in frequency between different observations, whereas in black-hole candidates the QPO coherency appears to be lower $(Q \sim 2-10)$ and the presence of a pair emerges only when a collection of observations is carefully analysed.

In Abramowicz \& Kluźniak (2001) and Kluźniak \& Abramowicz (2001), the idea of accretion disc resonance was proposed, as naturally incorporating pairs of frequencies occurring in a ratio of small integer numbers. This scheme predicts the observed frequency ratios in black-hole QPO sources should prefer the 3:2 ratio. It also suggests this could be understood if a non-linear coupling mechanism operates in a black-hole accretion disc, where strong-gravity effects are essential. Especially in those black-hole candidates where the high-frequency QPOs have been reported, they indeed occur very close to the ratio of small integer numbers, 3:2 in particular (Miller et al. 2001; Strohmayer 2001; Homan et al. 2004; Remillard et al. 2006; Maccarone \& Schnittman 2005). Nowadays, the original account can be viewed as a naïve model with internal resonance. Various realizations of this scheme have been examined in terms of accretion disc/torus oscillations (e.g. Abramowicz et al. 2003; Bursa et al. 2004; Kato 2004; Li \& Narayan 2004; Schnittman \& Rezzolla 2006; Zanotti et al. 2005).

So far, the "right" model has not yet been identified. However, it has been recognized that fruitful knowledge of the common properties of high-frequency QPOs can be gained by investigating a very general resonance scheme that very likely governs matter near a compact accreting body. To this aim, Abramowicz et al. (2003) examined the epicyclic resonances in a nearly-geodesic motion in strong gravity. By employing the method of multiple scales (Nayfeh \& Mook 1979), Rebusco (2004) and Horák (2004) demonstrate that the 3:2 resonance is indeed the most prominent one near horizon of a central black hole. Only certain frequency combinations are allowed, depending on the symmetries that the system exhibits, and only some of the allowed combinations have a chance of giving rise to a strong resonance.

In the present paper we pursue this approach further and find tracks that an axially symmetric system with two degrees 
of freedom, near resonance, should follow in the plane of energy (of the oscillations) versus radius (where the oscillations take place). We show different topologies of the phase space in a way that closely resembles the disturbing function method, familiar from the studies of the evolution of mean orbital elements in celestial mechanics (Kozai 1962; Lidov 1962). The analogy is very illuminating and provides a systematic way of distinguishing the topologically different states of the system. In particular, one can distinguish regions of phase space where the observed frequency ratio fluctuates around an exact rational number from those regions where this ratio always remains outside the resonance. Our model suggests that even black-hole twin QPOs should vary in frequency and should not stay at a firmly fixed frequency ratio, although the expected variation is very small - certainly less than what has been frequently reported in neutron-star binaries and what can be tested with the data available at present.

\section{A conservative system with two degrees of freedom}

\subsection{Non-linear terms in the governing equations}

Let us consider an oscillatory system with two degrees of freedom, which is described by coupled differential equations of the form

$$
\begin{aligned}
\ddot{\delta} \rho+\omega_{r}^{2} \delta \rho & =f_{\rho}(\delta \rho, \delta \theta, \dot{\delta \rho}, \dot{\delta} \theta), \\
\ddot{\delta} \theta+\omega_{\theta}^{2} \delta \theta & =f_{\theta}(\delta \rho, \delta \theta, \dot{\delta \rho}, \dot{\delta} \theta) .
\end{aligned}
$$

We assume that the right-hand functions are nonlinear (their Taylor expansions start with the second order) and that they are invariant under reflection of time. Clearly, these equations include the case of a nearly circular motion under the influence of a perturbing force: $\delta \rho$ and $\delta \theta$ are small deviations of the position, whereas $\omega_{r}(r)$ and $\omega_{\theta}(r)$ are the radial and vertical epicyclic frequencies along the circular orbit, $r=r_{0}, \theta=\pi / 2$ :

$\omega_{r}^{2}=\frac{\partial^{2} \mathcal{U}}{\partial r^{2}}, \quad \omega_{\theta}^{2}=\frac{1}{r_{0}^{2}}\left(\frac{\partial^{2} \mathcal{U}}{\partial \theta^{2}}\right)$,

where the effective potential is

$\mathcal{U}(r, \theta) \equiv \Phi(r, \theta)+\frac{\ell^{2}}{2 r^{2} \sin ^{2} \theta}$,

$\Phi(r, \theta)$ is the gravitational potential, for which axial symmetry and staticity will be assumed. These assumptions make our system qualitatively different from models requiring nonaxisymmetric perturbations.

As a generic example, let the total gravitational field be given as a superposition of the central potential of a spherical star, $\Phi_{\mathrm{s}}(r)$, plus an axisymmetric term $\Phi_{\mathrm{r}}(r, \theta)$,

$\Phi(r, \theta)=\Phi_{\mathrm{s}}(r)+\Phi_{\mathrm{r}}(r, \theta)$.

For the central field we assume the form

$\Phi_{\mathrm{s}}(r)=-\frac{G M}{\tilde{r}}$,

where we set $\tilde{r}=r$ or $\tilde{r}=r-R_{\mathrm{S}}$ (to adopt the Newtonian or the pseudo-Newtonian approximations; $R_{\mathrm{S}} \equiv 2 G M / c^{2}$ ). We assume a circular ring (mass $m$, radius $a$ ) as a source of the perturbing potential,

$\Phi_{\mathrm{r}}(r, \theta)=-\frac{2 G m}{\pi} \frac{K(k)}{B^{1 / 2}}$

where $K(k)$ is a complete elliptical integral of the first kind, $B(r, \theta) \equiv r^{2}+a^{2}+2 a r \sin \theta, k(r, \theta) \equiv 4 \operatorname{ar} \sin \theta / B(r)$.

At this point a remark is necessary regarding the interpretation of the potential (5)-(7): we conceive of it as a toy-model for strong-gravity effects and internal resonances that we seek in the system of a black hole and an accretion disc, but the origin of the perturbing potential $\Phi_{\mathrm{r}}(r, \theta)$ is not supposed to be the gravitational field of the accretion disc itself. Of course, the inner disc is not self-gravitating in black-hole binaries, and the ring is not introduced here with the aim of representing the accretion flow gravity. What we imagine is that hydrodynamic and magnetic forces are producing qualitative effects, which can be captured by the ring potential in our Eqs. (1), (2). On the other hand, our approach is rather general, and it is worth remembering that the same formalism can also be successfully applied in systems where the disc self-gravity plays a non-negligible role.

Also in general relativity, weakly perturbed (i.e. nearlygeodesic) motion of gas elements orbiting around a Schwarzschild black hole can be described by effective potential that contains a spherical term arising from the gravitational field of the central black hole, plus a perturbing term, which we assume is axially symmetric. Naturally, interpretation of the perturbing term is more complicated if one would like to derive its particular form from an exact solution of Einstein's equations. We do not want to enter into complications of a special model here, but see Letelier (2003), Karas et al. (2004), Semerák (2004), and references cited therein for a review and examples of spacetimes that contain a black hole and a gravitating ring in general relativity. Also, one may ask whether the adopted approach can comprehend frame-dragging effects of Kerr spacetime while the black hole rotation is considered as perturbation of the spherical field of a static non-rotating black hole. Even if a general answer to that question is affirmative, the Kerr metric has rather special properties concerning the integrability of the geodesic motion and the form of nonsphericity that quickly decays with radius. Our current opinion is such that the rotation-related effects of the Kerr metric cannot be viewed as the origin of the perturbation required for blackhole high-frequency twin QPOs.

The angular momentum of a test particle orbiting the centre on an equatorial circular orbit is

$\ell \equiv r^{3}\left(\frac{\partial \Phi}{\partial r}\right)=G M r\left[\frac{r^{2}}{\tilde{r}^{2}}+\mu r \frac{(r+a) E\left(k_{0}\right)+(r-a) K\left(k_{0}\right)}{\pi\left(r^{2}-a^{2}\right)}\right]$,

where $E(k)$ is a complete elliptical integral of the second kind and the right-hand terms are evaluated at the orbit radius, $k_{0} \equiv$ $k\left(r_{0}, \pi / 2\right), \mu \equiv m / M$. Figure 1 captures a typical curve of the angular momentum as a function of radius. The corresponding orbital frequency is

$\Omega^{2}(r)=\frac{G M}{r^{3}}\left[\frac{r^{2}}{\tilde{r}^{2}}+\mu r \frac{(r+a) E\left(k_{0}\right)+(r-a) K\left(k_{0}\right)}{\pi\left(r^{2}-a^{2}\right)}\right]$. 

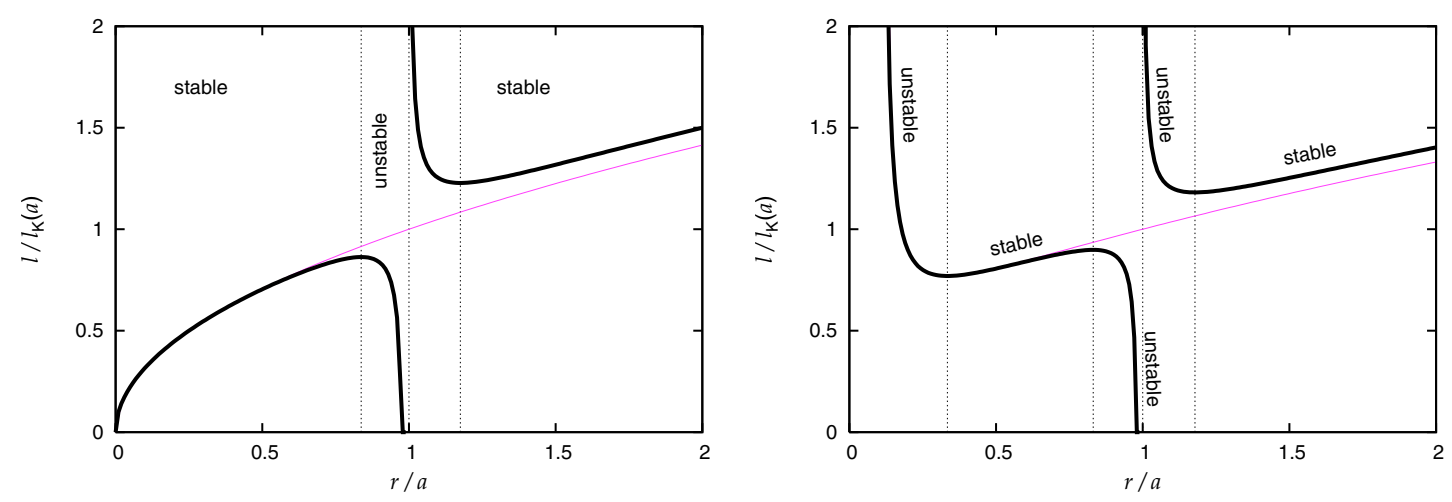

Fig. 1. Angular momentum $\ell(r)$ (thick line) of a test particle on a circular orbit in the combined gravitational field of a spherical body and a ring. The mass of the ring relative to the central mass is $\mu=0.1$, and the particle mass is set to unity. Left panel: the case of the Newtonian central field. Right panel: the pseudo-Newtonian case. Radius has been scaled with respect to the ring radius (here, $a=9 R_{\mathrm{S}}$ ), and the angular momentum has been scaled by the value of Keplerian angular momentum $\ell_{\mathrm{K}}(a)$. Keplerian angular momentum in the central field is also plotted (thin line). Circular orbits are Rayleigh unstable and the epicyclic approximation is inadequate in regions where the angular momentum decreases with radius.
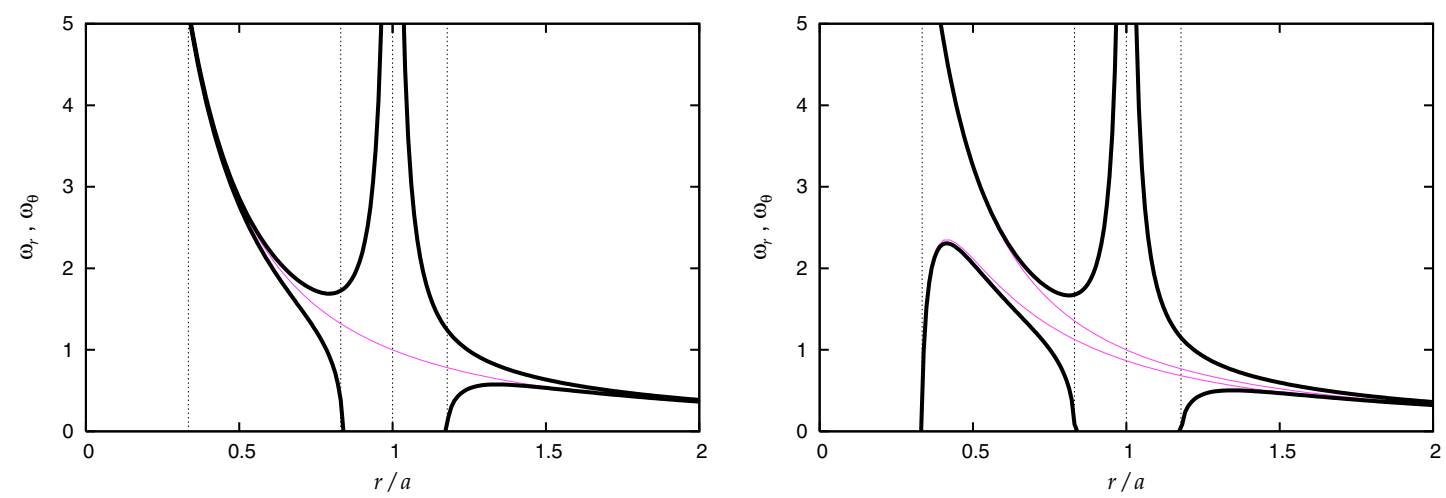

Fig. 2. The behaviour of epicyclic frequencies (thick lines; $\omega_{\theta}-t o p, \omega_{r}-$ bottom) provides basic clues to the origin of oscillations and the effects expected to occur in strong gravity. Left/right panels refer to the Newtonian/pseudo-Newtonian cases, as in the previous figure. Units of the ordinates are scaled by $\sqrt{G M / a^{3}}$ to make frequencies dimensionless. The frequencies in absence of the ring $(\mu=0)$ are also indicated for reference (thin lines; $\omega_{\theta}(r)=\omega_{r}(r)$ in the Newtonian case).

Equation (3) gives the epicyclic frequencies,

$\omega_{r}^{2}(r)=\frac{G M}{r^{3}}\left[\frac{r^{2}(3 \tilde{r}-2 r)}{\tilde{r}^{3}}+\frac{2 \mu}{\pi} \frac{(r-a)^{2} K\left(k_{0}\right)-a^{2} E\left(k_{0}\right)}{r^{2}(r-a)^{2}(r+a)}\right]$,

$\omega_{\theta}^{2}(r)=\frac{G M}{r^{3}}\left[\frac{r^{2}}{\tilde{r}^{2}}+\frac{2 \mu}{\pi} \frac{E\left(k_{0}\right)}{(r-a)^{2}(r+a)}\right]$.

The epicyclic frequencies as functions of radius are plotted in Fig. 2 (we drop the index " 0 " for the sake of brevity). The difference between the radial epicyclic frequency and the orbital frequency gives the shift of pericentre. The difference of vertical epicyclic and orbital frequencies gives the nodal precession.

Internal resonances can occur in the system (1)-(2). In order to capture this phenomenon, we carry out a multiple-scales expansion (Nayfeh \& Mook 1979) in the form

$\delta \rho(t, \epsilon)=\sum_{n=1}^{4} \epsilon^{n} \rho_{n}\left(T_{\mu}\right), \quad \delta \theta(t, \epsilon)=\sum_{n=1}^{4} \epsilon^{n} \theta_{n}\left(T_{\mu}\right)$,

where $T_{\mu}=\epsilon^{\mu} t$ are treated as independent time scales. We terminate the expansion at the fourth order $(\mu=0,1,2,3$; the number of time scales is the same as the order at which the expansions are truncated).
Time derivatives take the form of expansions

$\frac{\mathrm{d}}{\mathrm{d} t}=\sum_{\mu=0}^{4} \epsilon^{\mu} D_{\mu}, \quad \frac{\mathrm{d}^{2}}{\mathrm{~d} t^{2}}=\sum_{\mu=0}^{4} \sum_{v=0}^{4} \epsilon^{\mu+v} D_{\mu} D_{\nu}$

where $D_{\mu} \equiv \partial / \partial T_{\mu}$. The method tackles the governing equations in their general form.

As a specific example we can adopt an explicit form describing the orbital motion,

$\delta \ddot{\rho}+\omega_{r}^{2} \delta \rho=(1+\delta \rho) \delta \dot{\theta}^{2}-\left[\frac{1}{r} \frac{\partial \mathcal{U}}{\partial r}-\omega_{r}^{2} \delta \rho\right]$

$\delta \ddot{\theta}+\omega_{\theta}^{2} \delta \theta=-2 \frac{\delta \dot{\rho} \delta \dot{\theta}}{1+\delta \rho}-\left[\frac{1}{(1+\delta \rho)^{2}} \frac{\partial \mathcal{U}}{\partial \theta}-\omega_{\theta}^{2} \delta \theta\right]$.

Because Eqs. (14), (15) are conservative, the growth of energy in one mode must be balanced by the energy loss in the other mode. Close to resonance radii, where the two epicyclic frequencies are in a ratio of small integers, the periodic exchange of energy should occur at a more pronounced rate. Because amplitudes of the oscillations are connected with eccentricity and inclination, the solution alternates between an inclined, almost circular trajectory at certain stages and an eccentric, almost equatorial case at other time. We note that, in a non-linear 
system, the eigenfrequencies $\omega_{r}, \omega_{\theta}$ are expected to differ from the observed (i.e. predicted) frequencies, which can be revealed e.g. by Fourier analysis of data time series. The relevance of this fact for QPOs was first recognized by Abramowicz et al. (2003) and Rebusco (2004), when they discussed a model for Sco X-1. It was further employed by Horák et al. (2004), who suggested that a connection should exist between the highfrequency QPOs and normal-branch oscillations.

We expand the effective potential derivatives and the functions $f_{\rho}$ and $f_{\theta}$ into Taylor series, up to the fourth order about a circular orbit. The expansion provides many nonlinear terms containing various derivatives

$u_{i j} \equiv\left(\frac{\partial^{i+j} \mathcal{U}}{\partial r^{i} \partial \theta^{j}}\right)_{\left[r_{0}, \pi / 2\right]}$

By imposing constraints on the potential and its derivatives, we identify resonances that are expected in a particular system (and reject those resonances that cannot be realised). In the next subsection, formulation of these constraints is still kept completely general, and valid for the arbitrary form of $\mathcal{U}$. Only later, in Sect. 2.3, we come to our original motivation from the orbital motion around a black hole and employ the symmetry of the potential $\mathcal{U}$. We then consider the case of a potential that is symmetric with respect to the equatorial plane. This implies the condition $u_{i(2 k+1)}=0, k \in \mathcal{N}$, somewhat reducing the number of terms in the expansions.

Amplitudes of the oscillations are characterised by a small parameter: $\delta \rho \sim \epsilon, \delta \theta \sim \epsilon$. We impose the solvability constraints and seek a solution in the form (12). To this aim, we first write the explicit form of these constraints in different orders of approximation.

\subsection{Solvability constraints}

In the first order, we obtain equations describing two independent harmonic oscillators,

$\left(D_{0}^{2}+\omega_{r}^{2}\right) \rho_{1}=0, \quad\left(D_{0}^{2}+\omega_{\theta}^{2}\right) \theta_{1}=0$.

The solutions can be expressed in the form

$\rho_{1}=\widehat{A}_{\rho}+\widehat{A}_{-\rho}, \quad \theta_{1}=\widehat{A}_{\theta}+\widehat{A}_{-\theta}$,

where we denote $\widehat{A}_{x} \equiv A_{x} \mathrm{e}^{\mathrm{i} \omega_{x} T_{0}}, \widehat{A}_{-x}=A_{x}^{*} \mathrm{e}^{-\mathrm{i} \omega_{x} T_{0}}$ with $x=\rho$ or $\theta$. The complex amplitudes $A_{x}$ generally depend on slower scales, $T_{1}, T_{2}$, and $T_{3}$.

An algorithmic nature of the multiple-scales method allows us to determine the form of solvability conditions in a conservative system with two degrees of freedom. The conditions arise by eliminating the terms that are secular in the fastest variable, $T_{0}$. This constraint is imposed, because otherwise the solutions in the form of series would not converge uniformly. In fact, the reason the same number of scales is required as the order of approximation in the expansion (12) is that one secular term gets eliminated in each order, and therefore the functions $A_{x}\left(T_{\mu}\right)$ are determined by the same number of solvability conditions as the number of their variables.
Table 1. Possible resonances and secular terms in the second order of approximation. Only regular secular terms are present if $\omega_{\theta}$ and $\omega_{r}$ are outside resonance, as indicated in the left column. Subsequent lines refer to a system in 1:2 and 2:1 resonances, respectively. In each record corresponding to the resonances, the first/second row gives an expression for secular terms in radial/vertical oscillations. To simplify our notation we introduced $\Lambda_{\alpha} \equiv C_{\alpha}^{(\rho)}$ and $K_{\alpha} \equiv C_{\alpha}^{(\theta)}$.

\begin{tabular}{cl}
\hline \hline$\omega_{\theta}: \omega_{r}$ & \multicolumn{1}{c}{ Secular terms } \\
\hline $\begin{array}{c}\text { Outside } \\
\text { resonance }\end{array}$ & $-2 \mathrm{i} \omega_{r} D_{1} \widehat{A}_{\rho}$ \\
\hline $1: 2$ & $-2 \mathrm{i} \omega_{\theta} D_{1} \widehat{A}_{\theta}$ \\
& $-2 \mathrm{i} \omega_{r} D_{1} \widehat{D}_{1} \widehat{A_{\rho}}, K_{1001} \widehat{A}_{-\rho} \widehat{A}_{020} \widehat{A}_{\rho}^{2}$ \\
\hline $2: 1$ & $-2 \mathrm{i} \omega_{r} D_{1} \widehat{A}_{\rho}, K_{0002} \widehat{A}_{\theta}^{2}$ \\
& $-2 \mathrm{i} \omega_{\theta} D_{1} \widehat{A}_{\theta}, \Lambda_{0110} \widehat{A}_{\rho} \widehat{A}_{-\theta}$ \\
\hline
\end{tabular}

In the second order, the terms proportional to $\epsilon^{2}$ in the expanded left-hand side of the governing Eqs. (1), (2) are

$\left[\ddot{\delta} x+\omega_{x}^{2} x\right]_{2}=\left(D_{0}^{2}+\omega_{x}^{2}\right) x_{2}+2 \mathrm{i} \omega_{x} D_{1} \widehat{A}_{x}-2 \mathrm{i} \omega_{x} D_{1} \widehat{A}_{-x}$.

On the right-hand side, the second-order terms result from the expansion of the nonlinearity $f_{x}(\delta \rho, \delta \theta, \dot{\delta} \rho, \dot{\delta \theta})$ with $D_{0} \rho_{1}$ and $D_{0} \theta_{1}$ in place of $\dot{\delta \rho}$ and $\dot{\delta} \theta$, respectively. They can be expressed as linear combinations of quadratic terms constructed from $\widehat{A}_{ \pm \rho}$ and $\widehat{A}_{ \pm \theta}$ :

$\left[f_{x}(\delta \rho, \delta \theta, \dot{\delta \rho}, \dot{\delta \theta})\right]_{2}=\sum_{|\alpha|=2} C_{\alpha}^{(x)} \widehat{A}_{-\rho}^{\alpha_{1}} \widehat{A}_{\rho}^{\alpha_{2}} \widehat{A}_{-\theta}^{\alpha_{3}} \widehat{A}_{\theta}^{\alpha_{4}}$

where $\alpha=\left(\alpha_{1}, \ldots, \alpha_{4}\right)$ and $|\alpha|=\alpha_{1}+\ldots+\alpha_{4}$. The constants $C_{\alpha}^{(x)}$ are given by angular frequencies of $\omega_{x}$ and by coefficients of the Taylor expansion of $f_{x}$. Equating the right-hand sides of Eqs. (19), (20) we find

$$
\begin{aligned}
\left(D_{0}^{2}+\omega_{x}^{2}\right) x_{2}= & -2 \mathrm{i} \omega_{x} D_{1} \widehat{A}_{x}+2 \mathrm{i} \omega_{x} D_{1} \widehat{A}_{-x} \\
& +\sum_{|\alpha|=2} C_{\alpha}^{(x)} \widehat{A}_{-\rho}^{\alpha_{1}} \widehat{A}_{\rho}^{\alpha_{2}} \widehat{A}_{-\theta}^{\alpha_{3}} \widehat{A}_{\theta}^{\alpha_{4}} .
\end{aligned}
$$

The right-hand side of Eq. (21) contains one secular term independently of the eigenfrequencies $\omega_{r}$ and $\omega_{\theta}$. However, additional secular terms may appear in the resonance. For example, when $\omega_{r} \approx 2 \omega_{\theta}$, the terms proportional to $\widehat{A}_{\theta}^{2}$ in the $\rho$-equation $(x \rightarrow \rho)$ and $\widehat{A}_{\rho} \widehat{A}_{-\theta}$ in the $\theta$-equation $(x \rightarrow \theta)$ become secular and they should also be included in the solvability conditions. The similar situation happens when $\omega_{r} \approx \omega_{\theta} / 2$. These are internal resonances, which show a qualitatively different behaviour: the corresponding terms are secular only for special (resonant) combinations of $\omega_{r}$ and $\omega_{\theta}$, contrary to the terms that appear always and are referred to as regular secular terms. Possible resonances in the second order of approximation and the corresponding secular terms in Eq. (21) are listed in Table 1. Let us assume, for a moment, that the system is far from any resonance. Then

$D_{1} A_{x}=0$.

The frequencies and amplitudes are constant and the behaviour of the system is almost identical to what one finds in the linear 
Table 2. Possible resonances and secular terms in the third order of approximation. Individual records have a similar meaning as in Table 1.

\begin{tabular}{|c|c|}
\hline$\omega_{\theta}: \omega_{r}$ & Secular terms \\
\hline $\begin{array}{l}\text { Outside } \\
\text { resonance }\end{array}$ & $\begin{array}{lll}2 \mathrm{i} \omega_{r} D_{2} \widehat{A_{\rho}}, & K_{1200}\left|A_{\rho}\right|^{2} \widehat{A}_{\rho}, & K_{0111}\left|A_{\theta}^{2}\right| \widehat{A}_{\rho} \\
2 \mathrm{i} \omega_{\theta} D_{2} \widehat{A}_{\theta}, & \Lambda_{1101}\left|A_{\rho}\right|^{2} \widehat{A}_{\theta}, & \Lambda_{0012}\left|A_{\theta}^{2}\right| \widehat{A}_{\theta}\end{array}$ \\
\hline $1: 3$ & 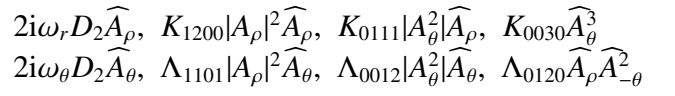 \\
\hline $1: 1$ & $\begin{array}{l}2 \mathrm{i} \omega_{r} D_{2} \widehat{A}_{\rho}, K_{1200}\left|A_{\rho}\right|^{2} \widehat{A}_{\rho}, \quad K_{0111}\left|A_{\theta}^{2}\right| \widehat{A}_{\rho}, \quad K_{1110}\left|A_{\rho}\right|^{2} \widehat{A}_{\theta}, \\
K_{0012}\left|A_{\theta}\right|^{2} \widehat{A}_{\theta}, K_{0210} \widehat{A}_{\rho}^{2} \widehat{A}_{-\theta}, K_{1002} \widehat{A}_{-\rho} \widehat{A}_{\theta}^{2} \\
2 \mathrm{i} \omega_{\theta} D_{2} \widehat{A}_{\theta}, \Lambda_{1101}\left|A_{\rho}\right|^{2} \widehat{A}_{\theta}, \Lambda_{0012}\left|A_{\theta}^{2}\right| \widehat{A}_{\theta}, \Lambda_{2100}\left|A_{\rho}\right|^{2} \widehat{A}_{\rho}, \\
\Lambda_{0021}\left|A_{\theta}\right|^{2} \widehat{A}_{\theta}, \Lambda 1002 \widehat{A}_{-\rho} \widehat{A}_{\theta}^{2}, \quad \Lambda_{0210} \widehat{A}_{\rho}^{2} \widehat{A}_{-\theta}\end{array}$ \\
\hline $3: 1$ & 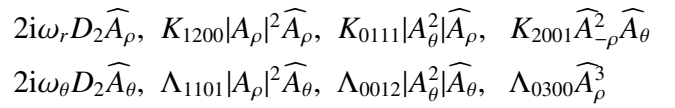 \\
\hline
\end{tabular}

approximation. The only difference is the presence of higher harmonics oscillating with frequencies $2 \omega_{r}, 2 \omega_{\theta}$, and $\left|\omega_{r} \pm \omega_{\theta}\right|$. They are given by a particular solution of Eq. (21) after eliminating the secular term,

$x_{2}=\sum_{|\alpha|=2} Q_{\alpha}^{(x)} \widehat{A}_{-\rho}^{\alpha_{1}} \widehat{A}_{\rho}^{\alpha_{2}} \widehat{A}_{-\theta}^{\alpha_{3}} \widehat{A}_{\theta}^{\alpha_{4}}$.

Under the assumption of time-reflection invariance, constants $Q_{\alpha}^{(x)}$ are real and their relation to constants $C_{\alpha}^{(x)}$ becomes obvious by substituting $x_{2}$ into Eq. (21). We find

$Q_{k l m n}^{(x)}=\frac{C_{k l m n}^{(x)}}{\omega_{x}^{2}-\left[(k-l) \omega_{r}+(m-n) \omega_{\theta}\right]^{2}}$.

In the third order, the discussion is analogous in many respects. The terms proportional to $\epsilon^{3}$, which appear on the left-hand side of the governing equations, are given by

$\left[\ddot{\delta x}+\omega_{x}^{2} x\right]_{3}=\left(D_{0}^{2}+\omega_{x}^{2}\right) x_{3}+2 \mathrm{i} \omega_{x} D_{2} \widehat{A}_{x}-2 \mathrm{i} \omega_{x} D_{2} \widehat{A}_{-x}$.

The terms containing $D_{1} x_{1}$ and $D_{1} x_{2}$ vanish as a consequence of the solvability condition (22). The right-hand side now contains cubic terms of the Taylor expansion. We obtain

$$
\begin{aligned}
\left(D_{0}^{2}+\omega_{x}^{2}\right) x_{3}= & -2 \mathrm{i} \omega_{x} D_{2} \widehat{A}_{x}+2 \mathrm{i} \omega_{x} D_{2} \widehat{A}_{-x} \\
& +\sum_{|\alpha|=3} C_{\alpha}^{(x)} \widehat{A}_{-\rho}^{\alpha_{1}} \widehat{A}_{\rho}^{\alpha_{2}} \widehat{A}_{-\theta}^{\alpha_{3}} \widehat{A}_{\theta}^{\alpha_{4}},
\end{aligned}
$$

where constants $C_{\alpha}^{(x)}$ are real. The secular terms are summarised in Table 2 together with resonances possible in the third order of approximation. Again, far from any resonance we eliminate the terms that are secular independent of $\omega_{r}, \omega_{\theta}$. The resulting solvability conditions take the form

$$
\begin{aligned}
D_{2} A_{\rho} & =-\frac{\mathrm{i}}{2 \omega_{r}}\left[K_{1200}\left|A_{\rho}\right|^{2}+K_{0111}\left|A_{\theta}\right|^{2}\right] A_{\rho}, \\
D_{2} A_{\theta} & =-\frac{\mathrm{i}}{2 \omega_{\theta}}\left[\Lambda_{1101}\left|A_{\rho}\right|^{2}+\Lambda_{0012}\left|A_{\theta}\right|^{2}\right] A_{\theta} .
\end{aligned}
$$

A particular solution of Eq. (26) is given by a linear combination of cubic terms constructed from $\widehat{A}_{ \pm \rho}$ and $\widehat{A}_{ \pm \theta}$,

\begin{tabular}{|c|c|}
\hline$\omega_{\theta}: \omega_{r}$ & Secular terms \\
\hline $\begin{array}{l}2 \mathrm{i} \omega_{r} D_{3} \widehat{A}_{\rho} \\
\text { resonance }\end{array}$ & $2 \mathrm{i} \omega_{\theta} D_{3} \widehat{A_{\theta}}$ \\
\hline $1: 4$ & $\begin{array}{ll}2 \mathrm{i} \omega_{r} D_{3} \widehat{A}_{\rho}, & K_{0004} \widehat{A}_{\theta}^{4} \\
2 \mathrm{i} \omega_{\theta} D_{3} \widehat{A}_{\theta}, & \Lambda_{0103} \widehat{A}_{\rho} \widehat{A}_{\theta}^{3}\end{array}$ \\
\hline $2: 3$ & $\begin{array}{ll}2 \mathrm{i} \omega_{r} D_{3} \widehat{A}_{\rho}, & K_{0130} \widehat{A}_{\rho} \widehat{A}_{-\theta}^{3} \\
2 \mathrm{i} \omega_{\theta} D_{3} \widehat{A}_{\theta}, & \Lambda_{0220} \widehat{A}_{\rho}^{2} \widehat{A}_{-\theta}^{2}\end{array}$ \\
\hline $3: 2$ & $\begin{array}{ll}2 \mathrm{i} \omega_{r} D_{3} \widehat{A}_{\rho}, & K_{2002} \widehat{A}_{-\rho}^{2} \widehat{A}_{\theta}^{2} \\
2 \mathrm{i} \omega_{\theta} D_{3} \widehat{A}_{\theta}, & \Lambda_{0310} \widehat{A}_{\rho}^{3} \widehat{A}_{-\theta}\end{array}$ \\
\hline $4: 1$ & $\begin{array}{ll}2 \mathrm{i} \omega_{r} D_{3} \widehat{A}_{\rho}, & K_{0301} \widehat{A}_{\rho}^{3} \widehat{A}_{\theta} \\
2 \mathrm{i} \omega_{\theta} D_{3} \widehat{A}_{\theta}, & \Lambda_{0400} A_{\rho}^{4}\end{array}$ \\
\hline
\end{tabular}

$x_{3}=\sum_{|\alpha|=3} Q_{\alpha}^{(3, x)} \widehat{A}_{-\rho}^{\alpha_{1}} \widehat{A}_{\rho}^{\alpha_{2}} \widehat{A}_{-\theta}^{\alpha_{3}} \widehat{A}_{\theta}^{\alpha_{4}}$,
Table 3. Possible resonances in the fourth order of approximation.

where all coefficients $Q_{\alpha}^{(3, x)}$ are now real.

Finally, in the fourth order of the approximation,

$\left[\ddot{\delta} x+\omega_{x}^{2} x\right]_{4}=\left(D_{0}^{2}+\omega_{x}^{2}\right) x_{3}+2 D_{3} D_{0} x_{1}+2 D_{0} D_{2} x_{2}$.

The operator $D_{0} D_{2}$ acts on $x_{2}$, given by Eq. (23). The resulting form is found by employing the solvability conditions (27) and (28):

$2 D_{0} D_{2} x_{2}=\omega_{x}^{2} \sum_{|\alpha|=4} J_{\alpha}^{(x)} \widehat{A}_{-\rho}^{\alpha_{1}} \widehat{A}_{\rho}^{\alpha_{2}} \widehat{A}_{-\theta}^{\alpha_{3}} \widehat{A}_{\theta}^{\alpha_{4}}$

where $J_{\alpha}^{(x)}$ are real constants. By expanding the right-hand side we arrive at the governing equation

$$
\begin{aligned}
{\left[D_{0}^{2}+\omega_{x}^{2}\right] x_{4}=} & -2 \mathrm{i} \omega_{x} D_{3} \widehat{A}_{x}+2 \mathrm{i} \omega_{x} D_{3} \widehat{A}_{-x} \\
& +\sum_{|\alpha|=4} C_{\alpha}^{(x)} \widehat{A}_{-\rho}^{\alpha_{1}} \widehat{A}_{\rho}^{\alpha_{2}} \widehat{A}_{-\theta}^{\alpha_{3}} \widehat{A}_{\theta}^{\alpha_{4}}
\end{aligned}
$$

with $C_{\alpha}^{(x)}$ real constants. Only one secular term independent of $\omega_{r}$ and $\omega_{\theta}$ appears on the right-hand side: $-2 \mathrm{i} \omega_{x} D_{3} \widehat{A}_{x}$. The sum only contains those terms that become secular near a resonance. These terms and the solvability conditions are listed in Table 3.

A notable feature of internal resonances $k: l$ is that $k \omega_{r}$ and $l \omega_{\theta}$ need not be infinitesimally close to each other, as might be expected from the linear analysis. Consider, for example, an internal resonance $1: 2$, i.e. $\omega_{\theta} \approx 2 \omega_{r}$. By eliminating the secular terms, we obtain solvability conditions (see Table 1)

$$
\begin{aligned}
-2 \mathrm{i} \omega_{r} D_{1} \widehat{A}_{\rho}+K_{1001} \widehat{A}_{-\rho} \widehat{A}_{\theta} & =0, \\
-2 \mathrm{i} \omega_{\theta} D_{1} \widehat{A}_{\theta}+\Lambda_{0200} \widehat{A}_{\rho}^{2} & =0 .
\end{aligned}
$$

In each of these equations the first term is regular, while the second term is nearly secular (resonant) one. The solvability conditions give us the long-term behaviour of the amplitudes and phases of oscillations. Suppose now that the system departs from the sharp ratio by small (first-order) deviations $\omega_{\theta}=2 \omega_{r}+\epsilon \sigma$, where $\sigma$ is the detuning parameter. The terms proportional to $\widehat{A}_{-\rho} \widehat{A}_{\theta}$ and $\widehat{A}_{\rho}^{2}$ still remain secular with respect to the variable $T_{0}$. This can be demonstrated from $\widehat{A}_{-\rho} \widehat{A}_{\theta}=A_{\rho}^{*} A_{\theta} \mathrm{e}^{\mathrm{i}\left(\omega_{\theta}-\omega_{r}\right) T_{0}}=A_{\rho}^{*} A_{\theta} \mathrm{e}^{\mathrm{i} \sigma T_{1}} \mathrm{e}^{\mathrm{i} \omega_{r} T_{0}}$. A similar relation holds for $\widehat{A}_{\rho}^{2}$. 


\subsection{Solution of the solvability constraints}

By comparing the coefficients with the same powers of $\epsilon$ on both sides of the Taylor-expanded governing Eqs. (1), (2), we obtain relations for functions $\rho_{i}\left(T_{j}\right)$ and $\theta_{i}\left(T_{j}\right)$ that can be solved successively. After rearranging to a "canonical" form,

$$
\begin{aligned}
& {\left[D_{0}^{2}+\omega_{r}^{2}\right] \rho_{n}=\sum K_{i j k l} \widehat{A}_{-\rho}^{i} \widehat{A}_{\rho}^{j} \widehat{A}_{-\theta}^{k} \widehat{A}_{\theta}^{t},} \\
& {\left[D_{0}^{2}+\omega_{\theta}^{2}\right] \theta_{n}=\sum \Lambda_{i j k l} \widehat{A}_{-\rho}^{i} \widehat{A}_{\rho}^{j} \widehat{A}_{-\theta}^{k} \widehat{A}_{\theta}^{7},}
\end{aligned}
$$

where $n$ is the order of approximation. In this way we identify constants $K_{i j k l}$ and $\Lambda_{i j k l}$. The studied gravitational potential is symmetric with respect to the equatorial plane; therefore, the series (35)-(36) cannot contain terms proportional to odd derivatives of the effective potential with respect to $\theta$. Hence, contrary to a general case, only specific resonances occur here: $\omega_{\theta}: \omega_{r}=1: 2,1: 1,3: 2$, and 1:4. These are all possible combinations that may occur within the given order of approximation (the first three cases were originally identified by Rebusco 2004, although she does not mention the fourth possible combination). A general argument of non-linear analysis suggests that the dominant resonances are those that correspond to ratios of small natural numbers, although not every conceivable resonant combination comes up in a given physical system. Indeed, it appears that the 3:2 ratio is the most important case where the high-frequency QPO pairs are debated, although the true role of this resonance has not yet been understood; see also the discussion in Bursa (2005) and Lasota (2005). Proceeding further to higher-order terms of the expansions reveals even more resonances, but these are expected to be very weak (recently various kinds of weird combinations have been examined by Török et al. 2005). Hereafter we concentrate on the first three combinations.

\section{The case of 1:2 resonance}

The solvability conditions take the form (cp. Table 1 and Horák 2004)

$$
\begin{aligned}
& D_{1} \widehat{A}_{\rho}=-\frac{\mathrm{i}}{2 \omega_{r}} K_{0002} \widehat{A}_{\theta}^{2}, \\
& D_{1} \widehat{A}_{\theta}=-\frac{\mathrm{i}}{2 \omega_{\theta}} \Lambda_{0110} \widehat{A}_{\rho} \widehat{A}_{-\theta} .
\end{aligned}
$$

The coefficients of the resonant terms are given by

$K_{0002}=-\omega_{\theta}^{2}-\frac{u_{12}}{2 r_{0}}, \quad \Lambda_{0110}=-2 \omega_{\theta}^{2}-\frac{u_{12}}{r_{0}}$,

satisfying a mutual relation $2 K_{0002}=\Lambda_{0110}$.

\section{The case of 1:1 resonance}

The solvability conditions for the first order, $D_{1} \widehat{A_{\rho}}=D_{1} \widehat{A_{\theta}}=0$, imply that the complex amplitudes $\widehat{A_{\rho}}$ and $\widehat{A_{\theta}}$ depend only on the second scale $T_{2}$. The 1:1 $\left(\omega_{r} \approx \omega_{\theta}\right)$ resonance is the only epicyclic resonance of the system with reflection symmetry, which occurs in the third order of approximation. The dependence on $T_{2}$ implies a slower behaviour. The solvability condi- tions are

$$
\begin{aligned}
D_{2} \widehat{A_{\rho}}= & -\frac{\mathrm{i}}{2 \omega_{r}}\left[K_{1200}\left|A_{\rho}\right|^{2} \widehat{A}_{\rho}\right. \\
& \left.+K_{0111}\left|A_{\theta}\right|^{2} \widehat{A}_{\rho}+K_{1002} \widehat{A}_{-\rho} \widehat{A}_{\theta}^{2}\right] \\
D_{2} \widehat{A_{\theta}=} & -\frac{\mathrm{i}}{2 \omega_{\theta}}\left[\Lambda_{1101}\left|A_{\rho}\right|^{2} \widehat{A}_{\theta}\right. \\
& \left.+\Lambda_{0012}\left|A_{\theta}\right|^{2} \widehat{A}_{\theta}+\Lambda_{0210} \widehat{A}_{\rho}^{2} \widehat{A}_{-\theta}\right]
\end{aligned}
$$

and the coefficients of the resonant terms are given by

$$
\begin{aligned}
K_{1200}= & r_{0}^{2}\left(\frac{5 u_{30}^{2}}{6 \omega_{\theta}}-\frac{1}{2} u_{40}\right), \\
K_{0111}= & \frac{1}{3}\left(-10 \omega_{\theta}^{2}+\frac{2 u_{12}^{2}}{r_{0}^{2} \omega_{\theta}^{2}}-3 u_{22}\right. \\
& \left.-6 r_{0} u_{30}+u_{12}\left[\frac{8}{r_{0}}+\frac{3 u_{30}}{\omega_{\theta}^{2}}\right]\right), \\
K_{1002}= & \frac{1}{6}\left(-6 \omega_{\theta}^{2}+\frac{6 u_{12}^{2}}{r_{0}^{2} \omega_{\theta}^{2}}-3 u_{22}-2 r_{0} u_{30}-\frac{u_{12} u_{30}}{\omega_{\theta}^{2}}\right), \\
\Lambda_{0012}= & -\frac{u_{04}}{2 r_{0}^{2}}-\frac{7 u_{12}}{6 r_{0}}+\frac{5 u_{12}^{2}}{6 r_{0}^{2} \omega_{\theta}^{2}}+\frac{10}{3} \omega_{\theta}^{2}, \\
\Lambda_{0210}= & K_{1002}, \\
\Lambda_{1101}= & K_{0111} .
\end{aligned}
$$

\section{The case of 3:2 resonance}

The solvability conditions involve both the third and the fourth orders, so the amplitudes $A_{\rho}, A_{\theta}$ are functions of both time scales $T_{3}$ and $T_{4}$. Eliminating of regular secular terms in the third order $\left(3 \omega_{r} \approx 2 \omega_{\theta}\right.$; see Table 2$)$ gives

$D_{2} \widehat{A}_{\rho}=-\frac{\mathrm{i}}{2 \omega_{r}}\left[K_{1200}\left|A_{\rho}\right|^{2} \widehat{A}_{\rho}+K_{0111}\left|A_{\theta}\right|^{2} \widehat{A}_{\rho}\right]$,
$D_{2} \widehat{A}_{\theta}=-\frac{\mathrm{i}}{2 \omega_{\theta}}\left[\Lambda_{1101}\left|A_{\rho}\right|^{2} \widehat{A}_{\theta}+\Lambda_{0012}\left|A_{\theta}\right|^{2} \widehat{A}_{\theta}\right]$,

with the coefficients

$$
\begin{aligned}
K_{1200}= & r_{0}^{2}\left(\frac{15 u_{30}^{2}}{8 \omega_{\theta}}-\frac{1}{2} u_{40}\right), \\
K_{0111}= & \frac{1}{4}\left(-15 \omega_{\theta}^{2}+\frac{9 u_{12}}{4 r_{0}^{2} \omega_{\theta}^{2}}-4 u_{22}\right. \\
& \left.-18 r_{0} u_{30}+9 u_{12}\left[\frac{1}{r_{0}}+\frac{u_{30}}{\omega_{\theta}^{2}}\right]\right), \\
\Lambda_{0012}= & -\frac{u_{04}}{2 r_{0}^{2}}+\frac{135}{64} \frac{u_{12}^{2}}{r_{0}^{2} \omega_{\theta}^{2}}-\frac{153}{16} \frac{u_{12}}{r_{0}}+\frac{135}{16} \omega_{\theta}^{2}, \\
\Lambda_{1101}= & K_{0111} .
\end{aligned}
$$

The elimination of the resonant terms gives the solvability condition in the fourth order (Table 3),

$D_{3} \widehat{A}_{\rho}=-\frac{\mathrm{i}}{2 \omega_{r}} K_{2002} \widehat{A}_{-\rho}^{2} \widehat{A}_{\theta}^{2}, \quad D_{3} \widehat{A}_{\theta}=-\frac{\mathrm{i}}{2 \omega_{\theta}} \Lambda_{0310} \widehat{A}_{\rho}^{3} \widehat{A}_{-\theta}$, 
where the resonant coefficients are

$$
\begin{aligned}
K_{2002}= & -\frac{15}{16} \omega_{\theta}^{2}+\frac{27}{32} \frac{u_{12}}{r_{0}}+\frac{135}{64} \frac{u_{12}^{2}}{r_{0}^{2} \omega_{\theta}^{2}}-\frac{243}{128} \frac{u_{12}^{3}}{r_{0}^{3} \omega_{\theta}^{4}} \\
& -\frac{9}{8} u_{22}+\frac{27}{16} \frac{u_{12} u_{22}}{r_{0} \omega_{\theta}^{2}}-\frac{27}{16} r_{0} u_{30}+\frac{81}{64} \frac{u_{12}^{2} u_{30}}{r_{0} \omega_{\theta}^{4}} \\
& -\frac{9}{16} \frac{r_{0} u_{22} u_{30}}{\omega_{\theta}^{2}}-\frac{81}{256} \frac{r_{0}^{2} u_{30}}{\omega_{\theta}^{2}}-\frac{81}{512} \frac{r_{0} u_{12} u_{30}^{2}}{\omega_{\theta}^{4}} \\
& -\frac{1}{4} r_{0} u_{30}-\frac{9}{64} r_{0}^{2} u_{40}-\frac{9}{128} \frac{r_{0} u_{12} u_{40}}{\omega_{\theta}^{2}},
\end{aligned}
$$

$\Lambda_{0310}=\frac{2}{3} K_{2002}$.

By introducing the detuning parameter,

$\sigma \equiv 3 \frac{\omega_{r}}{\omega_{\theta}}-2=\epsilon^{2} \tilde{\sigma}_{2}+\epsilon^{3} \tilde{\sigma}_{3}$,

the solvability conditions adopt the explicit form

$$
\begin{aligned}
& 2 \mathrm{i} \omega_{r} D_{3} A_{\rho}=K_{2002}\left(A_{\rho}^{\star}\right)^{2} A_{\theta}^{2} \mathrm{e}^{\mathrm{i}\left(\tilde{\sigma}_{2} T_{2}+\tilde{\sigma}_{3} T_{3}\right)}, \\
& 2 \mathrm{i} \omega_{\theta} D_{3} A_{\theta}=\Lambda_{0310} A_{\rho}^{3} A_{\theta}^{\star} \mathrm{e}^{\mathrm{i}\left(\tilde{\sigma}_{2} T_{2}+\tilde{\sigma}_{3} T_{3}\right)}, \\
& 2 \mathrm{i} \omega_{r} D_{2} A_{\rho}=\left[K_{1200}\left|A_{\rho}\right|^{2}+K_{0111}\left|A_{\theta}\right|^{2}\right] A_{\rho}, \\
& 2 \mathrm{i} \omega_{\theta} D_{2} A_{\theta}=\left[\Lambda_{1101}\left|A_{\rho}\right|^{2}+\Lambda_{0012}\left|A_{\theta}\right|^{2}\right] A_{\theta} .
\end{aligned}
$$

Finally, by substituting a polar form of complex amplitudes, $A_{\rho} \equiv \frac{1}{2} \tilde{a}_{\rho} \mathrm{e}^{\mathrm{i} \phi_{\rho}}$ and $A_{\theta} \equiv \frac{1}{2} \tilde{a}_{\theta} \mathrm{e}^{\mathrm{i} \phi_{\theta}}$, we get a set of eight equations governing the long-term behaviour of phases and amplitudes:

$D_{2} \tilde{a}_{\rho}=0, \quad D_{2} \tilde{a}_{\theta}=0$,

$D_{3} \tilde{a}_{\rho}=\frac{K_{2002}}{16 \omega_{r}} \tilde{a}_{\rho}^{2} \tilde{a}_{\theta}^{2} \sin \gamma, \quad D_{3} \tilde{a}_{\theta}=-\frac{\Lambda_{0310}}{16 \omega_{\theta}} \tilde{a}_{\rho}^{3} \tilde{a}_{\theta} \sin \gamma$,

$D_{2} \phi_{\rho}=-\frac{1}{8 \omega_{r}}\left[K_{1200} \tilde{a}_{\rho}^{2}+K_{0111} \tilde{a}_{\theta}^{2}\right]$,

$D_{2} \phi_{\theta}=-\frac{1}{8 \omega_{\theta}}\left[\Lambda_{1101} \tilde{a}_{\rho}^{2}+\Lambda_{0012} \tilde{a}_{\theta}^{2}\right]$,

$D_{3} \phi_{\rho}=-\frac{K_{2002}}{16 \omega_{r}} \tilde{a}_{\rho} \tilde{a}_{\theta}^{2} \cos \gamma, \quad D_{3} \phi_{\theta}=-\frac{\Lambda_{0310}}{16 \omega_{\theta}} \tilde{a}_{\rho}^{3} \cos \gamma$,

where the phase function has been introduced as $\gamma\left(T_{2}, T_{3}\right) \equiv$ $-\sigma_{2} T_{2}-\sigma_{3} T_{3}-3 \phi_{\rho}+2 \phi_{\theta}$. The amplitudes $\tilde{a}_{\rho}$ and $\tilde{a}_{\theta}$ of the oscillations vary slowly, because they only depend on the third time-scale $T_{3}$. Phases $\phi_{r}$ and $\phi_{\theta}$ of the oscillations evolve on both time scales $T_{2}$ and $T_{3}$.

\section{The system evolution near the 3:2 resonance}

\subsection{The integrals of motion}

The case of 3:2 resonance is particularly relevant for the highfrequency QPOs, both on observational and theoretical grounds (cf. Abramowicz \& Kluźniak 2001; Kluźniak et al. 2004, for arguments in favour of 3:2 ratio in high-frequency QPOs and for further references). We therefore discuss this case in more detail, although similar discussion could also be presented for other resonances (Horák 2005). Reintroducing single physical time $t$, the equations for the second and the third order can be

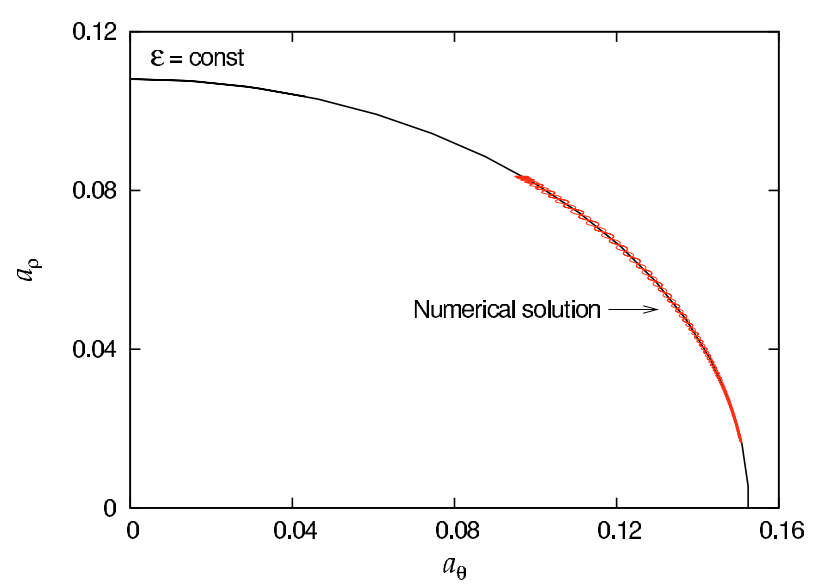

Fig. 3. Comparison between the analytical constraint $\mathcal{E}\left(a_{\rho}, a_{\theta}\right)=$ const. (an ellipse), derived in a multiple-scales approximation, and the corresponding exact (numerical) solution. The curly curve is the numerical solution of the oscillation amplitudes $a_{\rho}(t), a_{\theta}(t)$. The agreement between the two curves demonstrates that the accuracy of the approximation is satisfactory over the entire time span. See the text for details.

combined. Time derivatives are then given by $\mathrm{d} / \mathrm{d} t=\epsilon^{2} D_{2}+$ $\epsilon^{3} D_{3}$. Amplitudes and phases of the oscillations are governed by equations

$\dot{a}_{r}=\frac{1}{24} \beta \omega_{r} a_{r}^{2} a_{\theta}^{2} \sin \gamma$,

$\dot{a}_{\theta}=-\frac{1}{16} \beta \omega_{\theta} a_{r}^{3} a_{\theta} \sin \gamma$,

$\dot{\gamma}=-\sigma \omega_{\theta}+\frac{\omega_{\theta}}{4}\left[\mu_{r} a_{r}^{2}+\mu_{\theta} a_{\theta}^{2}+\frac{a_{r}}{2}\left(\alpha a_{\theta}^{2}-\beta a_{r}^{2}\right) \cos \gamma\right]$,

where $a_{\rho}=\epsilon \tilde{a}_{\rho}, a_{\theta}=\epsilon \tilde{a}_{\theta}$ and $\mu_{r}, \mu_{\theta}$ and $\beta$ are defined by relations $\Lambda_{0310}=\frac{2}{3} K_{2002}=\beta \omega_{\theta}^{2}, K_{1200}-\Lambda_{1101}=\omega_{r}^{2} \mu_{r}$, and $K_{0111}-\Lambda_{0012}=\omega_{\theta}^{2} \mu_{\theta}$. The amplitudes and phases are not mutually independent; Eqs. (66), (67) imply that the quantity

$\mathcal{E}=a_{\rho}^{2}+\frac{9}{4} a_{\theta}^{2}=$ const.

remains conserved during the system evolution. Clearly, $\mathcal{E}$ is proportional to the total energy of the oscillations. The existence of this integral is a general property of a conservative system. Naturally, it is not limited to the particular form of the perturbing potential (7), which we consider here, and it holds in Newtonian, pseudo-Newtonian, as well as general-relativity versions of the equations of motion (the pseudo-Newtonian case was examined, in detail, by Abramowicz et al. 2003 and Horák 2004). One can watch the accuracy to which $\mathcal{E}$ is conserved in order to verify the analytical approach against the exact numerical solution; we show such a comparison in Fig. 3 for the pseudo-Newtonian case of Abramowicz et al. (2003).

Equations (66), (67) can be merged into a single equation by introducing the following parameterization:

$a_{\rho}^{2}=\xi^{2} \mathcal{E}, \quad a_{\theta}^{2}=\frac{4}{9}\left(1-\xi^{2}\right) \mathcal{E}$.

Then the oscillations are described by two equations for $\xi(t)$ and $\gamma(t)$,

$\dot{\xi}=\frac{1}{16} \beta \omega_{\theta} \xi^{2}\left(1-\xi^{2}\right) \mathcal{E}^{3 / 2} \sin \gamma$, 


$$
\begin{aligned}
\dot{\gamma}= & -\sigma \omega_{\theta}+\frac{1}{4} \omega_{\theta} \mathcal{E}\left[\mu_{r} \xi^{2}\right. \\
& \left.+\frac{4}{9} \mu_{\theta}\left(1-\xi^{2}\right)+\frac{1}{4} \beta \xi\left(3-5 \xi^{2}\right) \mathcal{E}^{1 / 2} \cos \gamma\right],
\end{aligned}
$$

which satisfy the identity

$\dot{\xi} \mathrm{d} \gamma-\dot{\gamma} \mathrm{d} \xi=0$.

Substituting for $\dot{\xi}$ and $\dot{\gamma}$ from Eqs. (71)-(72), Eq. (73) implies that

$$
\begin{aligned}
\mathcal{F} \equiv & 8\left(1-\xi^{2}\right) \sigma+\mathcal{E}\left[\mu_{r} \xi^{4}-\frac{4}{9} \mu_{\theta}\left(1-\xi^{2}\right)^{2}\right] \\
& +\beta \mathcal{E}^{3 / 2} \xi^{3}\left(1-\xi^{2}\right) \cos \gamma
\end{aligned}
$$

is a second integral of motion. For a given value of energy $\mathcal{E}$, the system follows $\mathcal{F}=$ const curves. In other words, projection of the solution onto $(\gamma, \xi)$-plane satisfies

$\mathcal{F}(\gamma, \xi)=$ const.

This allows us to construct the two-dimensional phase-space sections in which the system evolution takes place.

\subsection{Stationary points and the phase-plane topology}

Stationary points are given by the condition $\dot{\xi}=\dot{\gamma}=0$. According to Eq. (71), the $\gamma$-coordinate of these points satisfies $\sin \gamma=0$, and therefore $\gamma=k \pi$ with $k$ an integer. Substituting $\dot{\gamma}=0$ and $\cos \gamma= \pm 1$ in Eq. (72), we find a cubic equation,

$-4 \sigma+\left[\mu_{r} \xi^{2}+\frac{4}{9} \mu_{\theta}\left(1-\xi^{2}\right)\right] \mathcal{E} \pm \beta \xi\left(3-5 \xi^{2}\right) \mathcal{E}^{3 / 2}=0$

The solution determines the $\xi$-coordinate of the stationary points. In the case of small oscillations $(\mathcal{E} \ll 1)$, the solution can be approximated by keeping only the terms up to the linear one in $\mathcal{E}$ in Eq. (76). We obtain

$\xi^{2}=\frac{9 \bar{\sigma}-\mu_{\theta}}{\frac{9}{4} \mu_{r}-\mu_{\theta}}$,

where $\bar{\sigma} \equiv \sigma / \mathcal{E}$. The first correction to this solution is of the order of $\mathcal{E}^{1 / 2}$. Deviations between $\xi$-coordinates of stationary points at odd and even multiples of $\pi$ are of the same order.

Solution (77) lies within the allowed range provided that $\frac{1}{4} \mu_{r} \lessgtr \bar{\sigma} \lessgtr \frac{1}{9} \mu_{\theta}$ with the denominator $D \equiv \frac{9}{4} \mu_{r}-\mu_{\theta} \lessgtr 0$. This can be expressed in terms of energy $\mathcal{E}$ : given the detuning parameter $\sigma$, stationary points appear in the $(\gamma, \xi)$ plane if the energy of oscillations satisfies

$9 \frac{\sigma}{\mu_{\theta}} \lessgtr \mathcal{E} \lessgtr 4 \frac{\sigma}{\mu_{r}}$ for $\quad D \gtrless 0$.

Examination of phase-plane topology near critical points leads to the equation

$\left(\frac{\partial \dot{\xi}}{\partial \xi}-\lambda\right)\left(\frac{\partial \dot{\gamma}}{\partial \gamma}-\lambda\right)-\frac{\partial \dot{\xi}}{\partial \gamma} \frac{\partial \dot{\gamma}}{\partial \xi}=0$

for eigenvalues $\lambda$ of the system of linearized Eqs. (71) and (72). Evaluating the partial derivatives at the critical point and keeping only the terms of the lowest order in $\mathcal{E}^{3 / 2}$, we obtain

$\lambda^{2}= \pm \frac{1}{72} \omega_{\theta}^{2} \beta \xi^{3}\left(1-\xi^{2}\right) D \mathcal{E}^{5 / 2}$

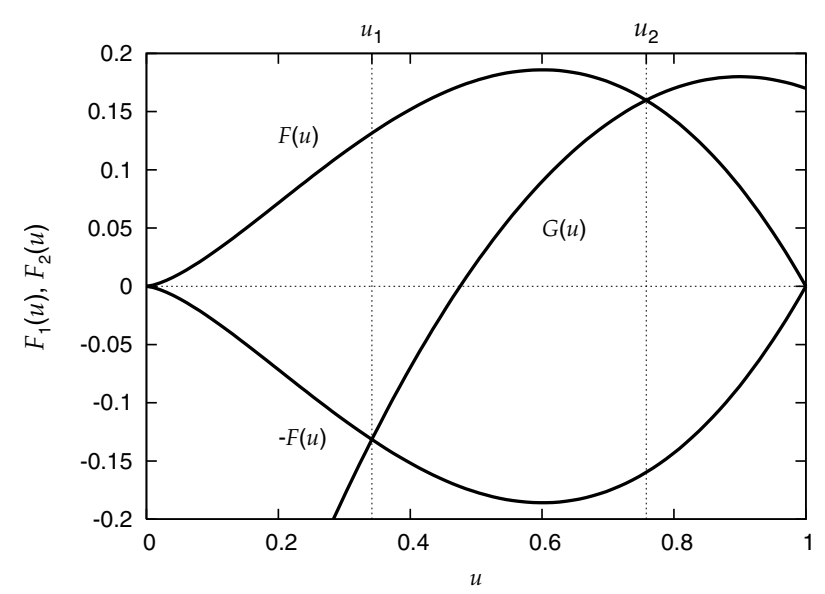

Fig. 4. The functions $F_{1} \equiv F(u), F_{2} \equiv-F(u)$, and $G(u)$ from Eq. (81). The system evolution is limited to the interval $\left\langle u_{1}, u_{2}\right\rangle$, where the condition $|F(u)| \geq|G(u)|$ is satisfied.

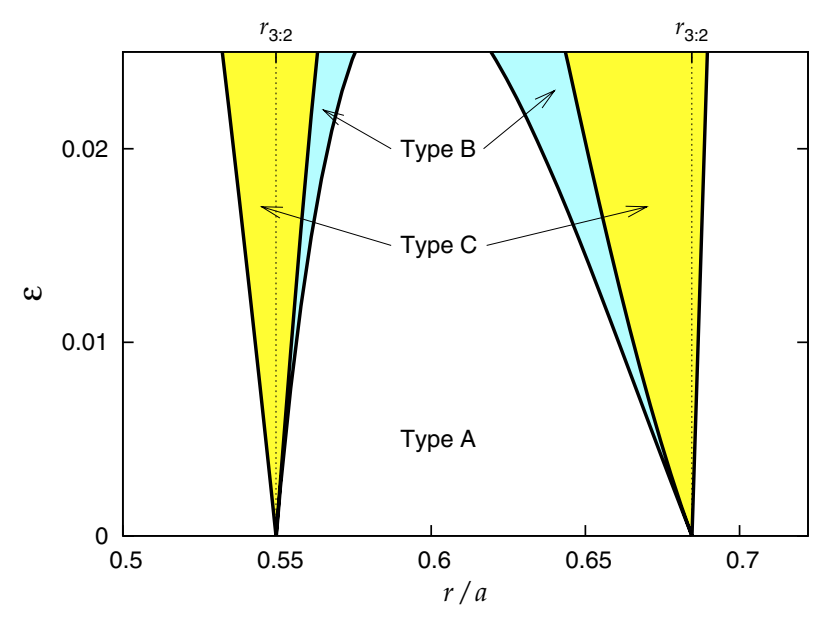

Fig. 5. The 3:2 inner resonances in the gravitational field of a spherical pseudo-Newtonian star and a ring $\left(a=9 R_{\mathrm{S}}, \mu=0.1\right)$. The regions of different phase-plane topology are identified on $(r, \mathcal{E})$-plane. Three types can be distinguished according to the number of critical points: $\mathrm{A}-$ no critical point (the system is far from resonance); $\mathrm{B}$ - one critical point; $\mathrm{C}-$ two critical points.

Examining the sign of $\lambda^{2}$ demonstrates that the critical points of central topology alternate with those of saddle topology.

\subsection{The time dependence}

The equation for $\xi(t)$ can be derived by combining Eqs. (71) and (74). Eliminating $\cos \gamma$, we arrive at the relation

$\mathcal{K} \dot{u}^{2}=F^{2}(u)-G^{2}(u)$,

where we have introduced a new variable $u(t) \equiv \xi^{2}$. The constant $\mathcal{K}$ and functions $F(u)$ and $G(u)$ are defined by

$$
\begin{aligned}
\mathcal{K} & \equiv \frac{1}{\mathcal{E}^{3 / 2}}\left(\frac{8}{\omega_{\theta} \beta}\right)^{2}, \\
F(u) & \equiv u^{3 / 2}(1-u) \\
G(u) & \equiv \frac{1}{\beta \mathcal{E}^{3 / 2}}\left[\mathcal{F}-8 \sigma(1-u)-\mu_{r} \mathcal{E} u^{2}+\frac{4}{9} \mu_{\theta} \mathcal{E}(1-u)^{2}\right] .
\end{aligned}
$$



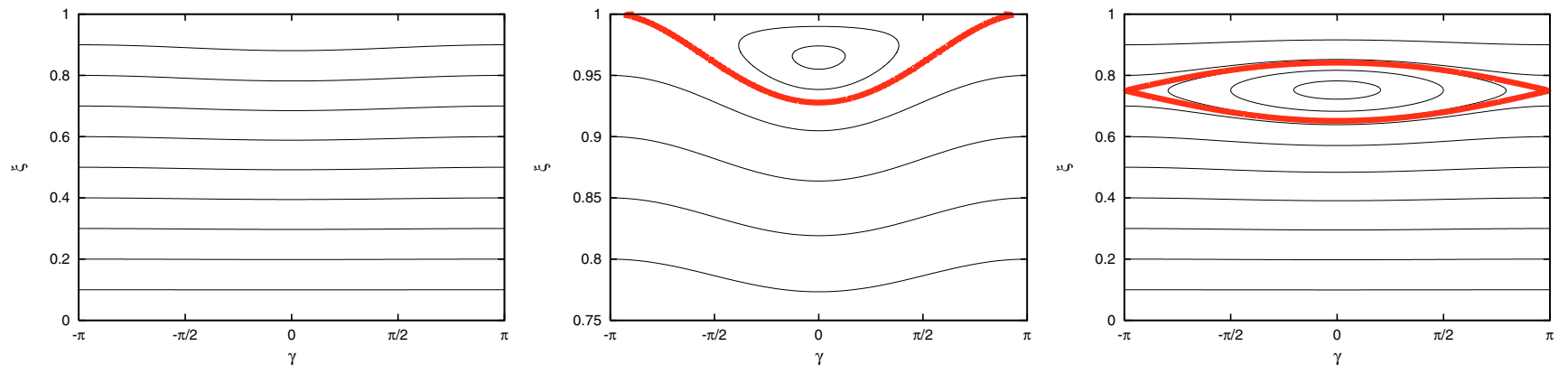

Fig. 6. Different topologies of the phase-space sections shown on the $(\xi, \gamma)$-plane. The system evolution follows the contour lines $\mathcal{F}(\xi, \gamma)=$ const. Their shape determines a possible range of the frequency change of the QPOs. The separatrix curve (thick line; cases B and C) divides the regions of circulating tracks from the regions of libration. Parameters and notation as in previous figure: A $(l e f t ; r=0.6 a, \mathcal{E}=0.02)$, $\mathrm{B}$ (middle; $r=0.65 a, \mathcal{E}=0.02), \mathrm{C}($ right $; r=0.55 a, \mathcal{E}=0.05)$.

The motion is allowed only for $\dot{u}^{2} \geq 0$, and so the condition $\pm F(u)=G(u)$ gives us two turning points, $u_{1}$ and $u_{2}$, between which the evolutionary path oscillates. The functions $\pm F(u)$ and $G(u)$ are plotted in Fig. 4 .

The period of energy exchange can be found by integrating Eq. (81):

$T=\frac{16}{\beta \omega_{\theta}} \mathcal{E}^{-3 / 2} \int_{u_{1}}^{u_{2}} \frac{\mathrm{d} u}{\sqrt{F^{2}(u)-G^{2}(u)}}$,

where $T$ can be roughly approximated as

$T \sim \frac{16 \pi}{\beta \omega_{\theta}} \mathcal{E}^{-3 / 2}$

Notice that this time-scale is longer than the period of individual oscillations, and it says how fast the system swaps itself between the radial and vertical oscillation modes. The simple estimate (86) is quite precise in most parts of the phase space, although it becomes inaccurate near stationary points, where the rate of energy exchange slows down.

Finally, we illustrate our results with a simple case, which we have already introduced at the beginning of the paper (Sect. 2.1): the gravitational field generated by a pseudoNewtonian star and a narrow circular ring. The resonant condition $\omega_{\theta} / \omega_{r}=3 / 2$ is now fulfilled at three different radii, two of them lying between the star and the ring, and the third one outside the ring. The resonances occurring at the first two radii are called the inner resonances, whereas the last is the outer resonance (not to be confused with "internal resonance", which all are). We restrict ourselves to the inner resonances, for which we find $\sigma, \mu_{r}, \mu_{\theta}$, and $\beta$ as functions of $r$. For a fixed radius, inequalities (78) give us the energy range of the oscillations. The result is shown in Fig. 5, where we identify three different phase-plane topologies in the $(r, \mathcal{E})$-section. These can be distinguished by the number of critical points and the shape of separatrices. The topology change is evident in Fig. 6, where two-dimensional plots are constructed for the integral of motion $\mathcal{F}(\xi, \gamma)$.

\subsection{Frequencies of the resonant oscillations}

Equations (63)-(65) give the shift of actual (observed) frequencies of oscillations, $\omega_{r}^{*}$ and $\omega_{\theta}^{*}$, with respect to the eigenfrequencies $\omega_{r}$ and $\omega_{\theta}$ :

$\omega_{r}^{*}=\omega_{r}+\dot{\phi}_{r}, \quad \omega_{\theta}^{*}=\omega_{\theta}+\dot{\phi}_{\theta}$.

These relations can be combined to find

$2 \omega_{\theta}^{*}-3 \omega_{r}^{*}=2 \omega_{\theta}-3 \omega_{r}+2 \dot{\phi}_{\theta}-3 \dot{\phi}_{r}=\dot{\gamma}$

The observed frequencies are in an exact 3:2 ratio if (and only if) the time-derivative of the phase function $\gamma$ vanishes. An immediate implication for the frequencies of stationary oscillations with constant amplitudes is that they stay in exact $3: 2$ ratio, even if the eigenfrequencies may depart from it. Outside stationary points, it is evident from Fig. 6 that $\dot{\gamma}=0$ represents turning points on libration tracks (those that are encircled by the separatrix curve). Hence, Eq. (88) discriminates between librating and circulating trajectories in the $(\gamma, \xi)$-plane. Circulating trajectories span the full range of $-\pi \leq \gamma<\pi$ and do not contain any turning point; $\dot{\gamma}$ remains nonzero and the twin frequencies never cross the exact $3: 2$ ratio in the region of circulation. On the other hand, there are two points $\dot{\gamma}=0$ on each librating trajectory. In such state the ratio of observed frequencies slowly fluctuates about 3:2.

\section{Conclusions}

We have discussed the resonance scheme for high-frequency QPOs via multiple-scales analysis, assuming an axisymmetric conservative system with two degrees of freedom. This approach provides useful insight into the general properties that are common to different conceivable mechanisms driving the oscillations, although it does not address the question of how the observed signal is actually formed and modulated. In our scenario, amplitudes and phases of the oscillations are mutually connected and follow tracks in the phase space with distinct topologies. The particular form was assumed to couple the oscillation modes via the non-spherical terms in the gravitational field of a ring. We consider this to be a toy-model for 
rather general behaviour that should take place in any system governed by equations of type (1)-(2).

We assumed the Newtonian (or the pseudo-Newtonian) description of the central gravitational field with perturbation by an aligned ring as an example. The adopted form is not essential for general conclusions. In fact, Eqs. (1)-(2) also cover the nearly-geodesic motion around a Schwarzschild black hole. Compared with the pseudo-Newtonian case, general relativity does not bring qualitatively new features, as long as the system is conservative; additional terms will arise in the expansions, which then translate to a slightly different value of the resonance radius and to a different duration of time intervals in physical units. A natural question arises as to whether the gravitational field of a rotating black hole could provide the perturbation required for the internal resonance in a surrounding disc. We considered this possibility, but it is unlikely that Kerr metric could suffice by itself. In the weak-field limit non-spherical terms seem to be incapable of creating separatrices in the phase-space sections discussed above, whereas in the full (exact, vacuum) Kerr metric the special mathematical properties of the spacetime ensure the integrability of the geodesic motion, and hence prevent the occurrence of internal resonances. Therefore, the problem of a specific mechanism launching and maintaining the oscillations remains unanswered.

Various options for the generalisation of our scheme could be motivated by papers of other authors who propose specific models including non-gravitational forces (see Pétri 2005, for a recent exposition of the problem and for references). As a nextstep towards an astrophysically realistic scheme, one should take dissipative and non-potential forces into account, as well as non-axisymmetric perturbations. These will allow our system to migrate across contours in the phase-plane and to undergo transitions when crossing separatrices. Such additional terms could also supplement the influence of external forcing and initiate the oscillations of the system. The internal resonance would then define the actual frequencies that are excited; this way the strong gravity unmasks itself.

Acknowledgements. V.K. appreciates fruitful discussions with participants at the Aspen Center for Physics workshop "Revealing Black Holes", and both of us thank for the hospitality of NORDITA (Copenhagen). We gratefully acknowledge the financial support of both the Czech Science Foundation (Refs. 205/06/P415 and 202/06/0041) and the Grant Agency of the Academy of Sciences (Ref. IAA300030510). The Astronomical Institute is operated under the project AV0Z10030501.

\section{References}

Abramowicz, M. A., Karas, V., Kluźniak, W., Lee, H., \& Rebusco, P. 2003, PASJ, 55, 467

Abramowicz, M. A., \& Kluźniak, W. 2001, A\&A, 374, L19

Barret, D., Kluźniak, W., Olive, J. F., Paltani, S., \& Skinner, G. K. 2005, MNRAS, 357, 1288

Bursa, M. 2005, in Processes in the Vicinity of Black Holes and Neutron Stars, Vol. 6/7, ed. S. Hledík, \& Z. Stuchlík, Silesian University, Opava, 39

Bursa, M., Abramowicz, M. A., Karas, V., \& Kluźniak, W. 2004, ApJ, 617, L45

Homan, J., Miller, J. M., Wijnands, R., et al. 2005, ApJ, 623, 383

Horák, J. 2004, in Processes in the Vicinity of Black Holes and Neutron Stars, Vol. 4/5, ed. S. Hledík, \& Z. Stuchlík, Silesian University, Opava, 91

Horák, J. 2005, Thesis, Charles University, Prague

Horák, J., Abramowicz, M. A., Karas, V., \& Kluźniak, W. 2004, PASJ, 56,819

Karas, V., Huré, J.-M., \& Semerák, O. 2004, CQG, 21, R1

Kato, S. 2004, PASJ, 56, 905

Kluźniak, W., \& Abramowicz, M. A. 2001, Acta Phys. Polonica B, 32,3605

Kluźniak, W., Abramowicz, M. A., Kato, S., Lee, W. H., \& Stergioulas, N. 2004, ApJ, 603, L89

Kozai, Y. 1962, AJ, 67, 591

Lasota, J.-P. 2005, Astron. Nachr., 326, 867

Letelier, P. S. 2003, Phys. Rev. D, 68, 104002

Li, Li-Xin, \& Narayan, R. 2004, ApJ, 601, 414

Lidov, M. L. 1962, Planetary and Space Sci., 9, 719

Maccarone, T. J., \& Schnittman, J. D. 2005, MNRAS, 357, 12

McClintock, J. E, \& Remillard, R. A. 2006, in Compact Stellar Xray Sources, ed. W. H. G. Lewin, \& M. van der Klis (Cambridge: Cambridge University Press)

Miller, J. M., Wijnands, R., Homan, J., et al. 2001, ApJ, 563, 928

Nayfeh, A. H., \& Mook, D. T. 1979, Nonlinear oscillations (New York: Wiley-Interscience)

Pétri, J. 2005, A\&A, 439, 443

Rebusco, P. 2004, PASJ, 56, 553

Remillard, R. A., McClintock, J. E., Orosz, J. E., \& Levine, A. M. 2006, ApJ, 637, 1002

Schnittman, J. D., \& Rezzolla, R. 2006, ApJ, 637, L113

Semerák, O. 2004, CQG, 21, 2203

Strohmayer, T. E. 2001, ApJ, 552, L49

Török, G., Abramowicz, M. A., Kluźniak, W., \& Stuchlík, Z. 2005, A\&A, 436, 1

van der Klis, M. 2006, in Compact Stellar X-ray sources, ed. W. H. G. Lewin, \& M. van der Klis (Cambridge: Cambridge University Press)

Zanotti, O., Font, J. A., Rezzolla, L., \& Montero, P. J. 2005, MNRAS, 356,1371 